\title{
História natural e biologia reprodutiva de marsupiais no Pantanal, Mato Grosso, Brasil
}

\author{
Mônica Aragona ${ }^{1,3} \&$ Jader Marinho-Filho ${ }^{2}$
}

\begin{abstract}
${ }^{1}$ Núcleo de Estudos Ecológicos do Pantanal, Universidade Federal de Mato Grosso. 78060-900 Cuiabá, Mato Grosso, Brasil. ${ }^{2}$ Departamento de Zoologia, Universidade de Brasília. 70910-900 Brasília, Distrito Federal, Brasil. E-mail: jmarinho@unb.br

${ }^{3}$ Corresponding Author. Rua Augusto dos Anjos 2, Santa Cruz, 78068-230 Cuiabá, Mato Grosso, Brasil.

E-mail: moaragona@ig.com.br
\end{abstract}

\begin{abstract}
Natural history and reproductive biology of marsupials from Pantanal, Mato Grosso, Brazil. Three forested habitats at Pantanal of Poconé (Mato Grosso) were sampled during 17 months, with an effort of 38,635 trapnights, resulting in a $6.3 \%$ of trapping success. Six marsupial and eight rodent species were recorded. Natural history, reproductive biology, behavior and habitat use are described for six marsupial species from the Pantanal floodplain: Caluromys philander (Linnaeus, 1758), Didelphis albiventris Lund, 1840, Gracilinanus agilis (Burmeister, 1854), Micoureus demerarae (Thomas, 1905), Monodelphis domestica (Wagner, 1842) and Philander opossum (Linnaeus, 1758). Micoureus demerarae was the species with the highest number of captured individuals $(n=240)$ and with the highest number of capture events $(n=1287)$. Reproduction of most species begins at the end of the dry season, being intensified during the wet season. The flooded forest locally known as 'landis' showed the highest marsupial species richness (six species), whereas the forests that are never flooded, locally known as 'cordilheiras', and the seasonally flooded forest, locally known as 'cambarazais', showed the lowest species richness, with three species each.
\end{abstract}

KEY WORDS. Habitat use; Pantanal floodplain.

Localizado na fronteira entre Brasil, Paraguai e Bolívia, o Pantanal é a maior planície intermitente e sazonalmente inundada do mundo. O pulso de inundação - a flutuação anual do nível da água - regula os processos ecológicos ali ocorrentes (Junk \& DA SiLva 1999), gerando uma periodicidade nas drásticas mudanças das condições ambientais dos habitats. A flora e a fauna da planície pantaneira são oriundas dos biomas adjacentes (Cerrado, Chaco, Mata Atlântica e Amazônia) e apresentam baixas taxas de endemismos (BRASIL 1997, JUNK \& DA Silva 1999, DA SiLva et al. 2001).

Atualmente 55 espécies de marsupiais são descritas para o Brasil, representando cerca de $8,4 \%$ das 652 espécies de mamíferos nativos, registradas e reconhecidas neste país (Reis et al. 2006). Para o Pantanal, 14 espécies de marsupiais são conhecidas. Treze delas ocorrem na borda, das quais seis também ocorrem na planície pantaneira, sujeita a alagamento. Uma espécie, Micoureus demerarae (Thomas, 1905) (= M. cinereus), está descrita apenas para a planície (Rodrigues et al. 2005). Das sete espécies descritas para a planície, cinco são abordadas neste estudo, além de Caluromys philander (Linnaeus, 1758), que só era descrita fora da planície. Todas são de ampla distribuição geográfica (EISENBERG \& REDFORD 1999) e a literatura acerca da história natural destas espécies é oriunda de diversos biomas, exceto do Pantanal. Informações sobre a biologia reprodutiva, dieta, comportamento e uso de habitat das espécies de marsupiais que ocorrem na planí- cie pantaneira podem ser encontrados, entre outros, em FonseCA \& KierulfF (1989) e Stallings (1989) para a Mata Atlântica, STreilein (1982 a, b) e Bergallo \& Cerqueira (1994) para a Caatinga, Alho et al. (1986) e Mares et al. (1989) para o Cerrado e PATton et al. (2000) e Voss et al. (2001) para a Amazônia.

Com o intuito de fortalecer o conhecimento acerca da história natural de marsupiais, este trabalho tem como objetivo descrever alguns aspectos da biologia reprodutiva, comportamento e uso de habitats de marsupiais no Pantanal matogrossense.

\section{MATERIAL E MÉTODOS}

Este trabalho foi desenvolvido na Fazenda Aparecida, Lo-

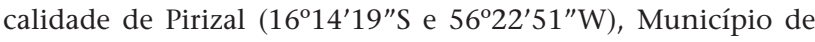
Nossa Senhora do Livramento, Pantanal de Poconé, Mato Grosso. Os marsupiais foram estudados em nove grades amostrais de três fisionomias florestais características da região do Pantanal de Poconé: cambarazal, cordilheira e landi. As coletas ocorreram mensalmente entre fevereiro de 2006 e agosto de 2007, excetuando os meses de janeiro e abril de 2007, totalizando 17 meses. Três estações foram definidas: cheia, entre dezembro e março; vazante, entre abril e julho; e seca, entre agosto e novembro.

Os cambarazais são formações monodominantes de Vochysia divergens Pohl. (Vochysiaceae), a qual é localmente denominada de Cambará. Esta é uma espécie pioneira, cuja distribuição está relacionada às áreas periodicamente inundáveis 
do Pantanal, espalhando-se rapidamente pelos campos naturais e manejados, formando extensos estandes (Prance \& Schaller 1982, Arieira \& Nunes da Cunha 2006). Apresenta alta taxa de crescimento sob intensa luminosidade, tolerância a alagamento prolongado e grande produção de sementes hidroanemocóricas. Suas populações são reduzidas pelo efeito de períodos plurianuais de grandes secas e aumentadas em grandes cheias (Nunes da CunHa \& Junk 2004).

Cordilheiras são extensas deposições de sedimentos fluviais nas bordas de leitos fósseis de rios, sendo formações estreitas, contínuas e sinuosas ao longo da paisagem (NuNES DA CunHa et al. 2006). Apresentam vegetação arbórea densa, predominando espécies do cerrado, com deciduidade e floração nos meses de julho e agosto. Trata-se de uma vegetação bastante heterogênea, com variações sensíveis na fisionomia e no estrato herbáceo-arbustivo, apresentando duas fisionomias mais comuns: uma formação densa, de até $2 \mathrm{~m}$ de altura, oferecendo grande dificuldade de acesso à região central da cordilheira, devido à grande abundância de gravatás (Bromelia balansae Mez - Bromeliaceae); e outra que se caracteriza pela presença de guiné (Petiveria tetrandra Gomez - Phytolaccaceae) e estrato arbóreo caracterizado por arvoretas da 5-6 m de altura. Árvores de grande porte, com até $30 \mathrm{~m}$ de altura, ocorrem na porção central da cordilheira (NunEs dA CunHa et al. 2006). Topograficamente, as cordilheiras estão em relevo positivo e não estão sujeitas ao alagamento.

Os landis são corredores de vegetação situados em relevo negativo, nas depressões de escoamento das águas, nos períodos de cheia e vazante. São florestas sempre verdes, de cerca de $8 \mathrm{~m}$ de altura, caracterizadas pela presença das espécies de Leiteiro (Mabea sp. - Euphorbiaceae e Alchornea discolor Poepp. Euphorbiaceae), Pimenteira (Licania parvifolia Huber Chrysobalanaceae) e Guanandi (Calophyllum brasiliense Camb. - Clusiaceae) (Nunes da Cunha et al. 2007).

Nove grades amostrais foram instaladas, três réplicas em cada tipo de habitat, em manchas distintas de vegetação. Cada grade era composta de 100 pontos amostrais (10 x 10) distantes $15 \mathrm{~m}$ entre si. Algumas áreas não permitiram a manutenção deste desenho $(10 \times 10)$ e foram ajustadas no campo, de acordo com a configuração encontrada. As nove grades tiveram a primeira linha de pontos de armadilhamento nos primeiros 5 m dentro da fisionomia florestal em relação à transição com o campo.

Dois modelos de armadilhas de captura viva, tipo sherman (9,5 × $8 \times 25 \mathrm{~cm}$, doravante referidas apenas como shermans) e gaiola de gancho $(16,5 \times 16,5 \times 35 \mathrm{~cm}$, doravante referidas apenas como gaiolas), foram utilizadas. Um pedaço de fruta, banana nas shermans e abacaxi nas gaiolas, e uma mistura de pasta de amendoim, fubá e sardinha foram usados como iscas. As armadilhas foram intercaladas quanto ao tipo e posição (solo e alto) por toda a grade e ao longo do tempo, de modo que um tipo em determinada posição só se repetisse em um mesmo ponto a cada quatro meses. As armadilhas do alto foram amarradas, com elásticos, a galhos, troncos ou lianas entre 1,0 e 2,5 m do solo, dentro de um raio de até $4 \mathrm{~m}$ em relação ao ponto, em função da disponibilidade de um apoio eficiente.

Apenas as grades das cordilheiras puderam ter os 100 pontos amostrados ao longo de todo o estudo (100 a-n), enquanto que as grades dos landis e cambarazais somente tiveram os 100 pontos amostrados se o solo estivesse disponível (100 a-n); na presença de lâmina d'água apenas os pontos do alto foram amostrados (50 a-n). Cada grade foi amostrada por três noites consecutivas (exceto em maio de 2006, com apenas duas noites), sendo verificadas todos os dias de manhã. Os animais capturados foram manipulados sem o uso de anestésicos e foram soltos no ponto e posição de sua captura. As armadilhas eram então imediatamente repostas em sua posição na grade.

Cada indivíduo capturado pela primeira vez foi marcado com um brinco numerado e as seguintes informações foram coletadas: espécie; sexo; classe etária (juvenil, sub-adulto e adulto; MACEDo et al. 2006); estádio reprodutivo das fêmeas (prenha, lactante e não reprodutiva); peso (g); medidas ( $\mathrm{mm}$ ) do corpo, cauda, cabeça, orelha direita, pata posterior direita com e sem unha; grade e ponto da grade; data, dia de armadilhamento (primeiro a terceiro) e estação (cheia, vazante e seca); tipo e posição da armadilha e observações comportamentais e ecológicas. A biometria, exceto o peso, foi realizada somente na primeira captura sendo as demais informações registradas nos casos de recaptura. O estádio reprodutivo somente foi avaliado para fêmeas, consideradas em período reprodutivo a partir de modificações em seu ventre: mudança da coloração pálida para um marrom-alaranjado, redução de pêlos e inchaço das tetas, além da presença de recém-nascidos no marsúpio ou fixados nas tetas. As shermans com capturas eram abertas no campo para a coleta de fezes (dados não analisados) e ao final da campanha de cada mês todas as shermans eram abertas e lavadas.

A razão sexual é dada pelo número de machos dividido pelo número de fêmeas. O cálculo do tempo de permanência foi feito considerando-se o número de meses entre as datas da primeira e da última captura de cada indivíduo. Para o cálculo do tempo de permanência médio (média aritmética dos tempos de permanência dos indivíduos), foram excluídos os indivíduos que morreram e os que foram capturados na última campanha.

Foi utilizado teste de Qui-quadrado (ZAR 1999) para comparações de diferenças nas capturas entre habitats, estações, tipo e posição das armadilhas, sendo as proporções ajustadas em função do número de armadilhas instaladas em cada comparação. O Programa MapSource 5.2 foi utilizado para o cálculo de distância e áreas.

Antes do início deste estudo, foram realizadas coletas para a preparação de uma coleção de referência (Licença 063/05 GEREX-I/MT; Doc. IBAMA: 02013.002797/05-89). Os espécimes coletados foram identificados no Museu de Zoologia da Universidade de São Paulo e posteriormente depositados na Coleção de Vertebrados da Universidade Federal de Mato Grosso. 


\section{RESULTADOS}

Um esforço de 38.635 armadilhas-noite (a-n) foi realizado e 729 indivíduos de 14 espécies foram capturados, sendo seis marsupiais e oito roedores (Tab. I). Foram registrados 2448 eventos de captura (primeira captura e recapturas), representando um sucesso total de captura de 6,3\%. Micoureus demerarae foi a espécie com o maior número de indivíduos capturados e com o maior número de eventos de captura, representando 32,9 e $52,6 \%$, respectivamente (Tab. I).

Tanto as cordilheiras como os cambarazais apresentam três espécies de marsupiais, sendo Monodelphis domestica (Wagner, 1842), uma espécie terrestre sem adaptações para hábito trepador, substituída por Philander opossum (Linnaeus, 1758) nos habitats alagáveis. Os landis apresentaram a maior riqueza, apesar da dominância de $M$. demerarae (Tab. II).

Apenas dois indivíduos de C. philander foram capturados, ambos no alto e em áreas de landis. Um macho sub-adulto, em sherman, na cheia de 2006 e uma fêmea adulta, com pelo menos quatro filhotes no marsúpio, em gaiola, na seca de 2006.

Apenas uma fêmea de $D$. albiventris Lund, 1840, sub-adulta e não reprodutiva, foi capturada em gaiola instalada no alto em uma área de landi, após onze meses de armadilhamento, na cheia de 2007.
Tabela II. Número de indivíduos de marsupiais capturados em cada fisionomia.

\begin{tabular}{lccc}
\hline \multicolumn{1}{c}{ Espécies } & Cambarazais & Cordilheiras & Landis \\
\hline C. philander & 0 & 0 & 2 \\
D. albiventris & 0 & 0 & 1 \\
G. agilis & 45 & 21 & 12 \\
M. demerarae & 27 & 87 & 127 \\
M. domestica & 0 & 61 & 2 \\
P. opossum & 20 & 0 & 7 \\
\hline Total & 92 & 169 & 151 \\
\hline
\end{tabular}

* Subtrair um indivíduo que mudou de uma grade de cordilheira para uma grade de cambarazal.

Foram capturados 78 indivíduos de Gracilinanus agilis (Burmeister, 1854) em 224 eventos de captura. Ocorreu nas três fisionomias e somente em uma área de landi não foi capturada. Foram significativamente mais capturados nos cambarazais $\left(\chi^{2}=54,92 ; \mathrm{gl}=2 ; \mathrm{p}<0,01\right)$ e durante a seca $\left(\chi^{2}=46,01 ; \mathrm{gl}=2\right.$; $\mathrm{p}<0,01$; Fig. 1) e em shermans no alto $\left(\chi^{2}=699,61 ; \mathrm{gl}=3\right.$; $\mathrm{p}<$ 0,001) para esforço de 100 a-n.

Tabela I. Resultado total do armadilhamento, para as nove grades de captura .

\begin{tabular}{|c|c|c|c|c|}
\hline Espécies & $\begin{array}{c}\text { Número de primeiras } \\
\text { capturas }\end{array}$ & \% do total de indivíduos & $\begin{array}{l}\text { Número total de } \\
\text { capturas }\end{array}$ & $\%$ do total de captura \\
\hline \multicolumn{5}{|l|}{ Marsupialia } \\
\hline Caluromys philander & 2 & 0,27 & 2 & 0,08 \\
\hline Didelphis albiventris & 1 & 0,14 & 1 & 0,04 \\
\hline Gracilinanus agilis & 78 & 10,70 & 224 & 9,15 \\
\hline Micoureus demerarae & 240 & 32,92 & 1287 & 52,57 \\
\hline Monodelphis domestica & 63 & 8,64 & 150 & 6,13 \\
\hline Philander opossum & 27 & 3,70 & 47 & 1,92 \\
\hline Sub-total & 411 & 56,37 & 1711 & 69,89 \\
\hline \multicolumn{5}{|l|}{ Rodentia } \\
\hline Calomys cf. callosus & 28 & 3,83 & 49 & 2,00 \\
\hline Holochilus sciureus & 1 & 0,14 & 1 & 0,04 \\
\hline Hylaeamys megacephalus & 58 & 7,96 & 79 & 3,23 \\
\hline Necromys lasiurus & 2 & 0,27 & 2 & 0,08 \\
\hline Oecomys mamorae & 29 & 3,98 & 89 & 3,64 \\
\hline Oecomys roberti & 127 & 17,42 & 301 & 12,30 \\
\hline Oligoryzomys cf. nigripes & 1 & 0,14 & 1 & 0,04 \\
\hline Thrichomys pachyurus & 72 & 9,88 & 215 & 8,78 \\
\hline Sub-total & 318 & 43,63 & 737 & 30,11 \\
\hline Total & 729 & & 2448 & \\
\hline
\end{tabular}




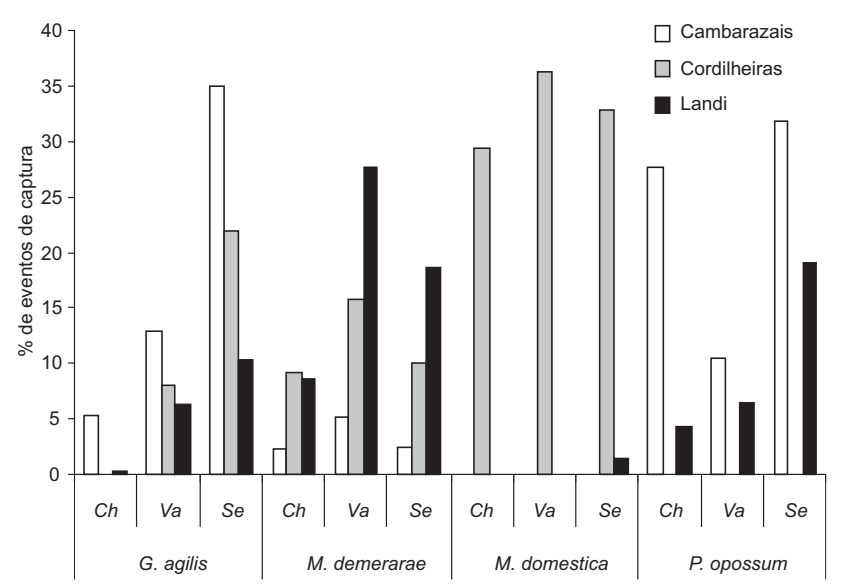

Figura 1. Freqüência relativa dos eventos de captura para cada espécie em cada da fisionomia na cheia (Ch), vazante (Va) e seca (Se), a partir dos dados mensais dos anos de 2006 e 2007.

A razão sexual total foi de 2,3:1 (54 machos e 24 fêmeas), sendo de 2:1 nas cordilheiras e landis e de 2,5:1 nos cambarazais.

Dentre as fêmeas, 14 foram capturadas uma única vez ou tiveram apenas uma recaptura no mesmo mês de sua primeira captura. Desconsiderando as recapturas ocorridas em um mesmo mês, foram feitos 51 registros de informação reprodutiva referentes a dez fêmeas; nenhum registro de atividade reprodutiva foi feito para sub-adultas. Gracilinanus agilis apresenta atividade reprodutiva o ano todo, sendo o investimento reprodutivo intensificado entre setembro e março (Fig. 2). Uma fêmea de $G$. agilis foi monitorada de agosto a novembro de 2006. Na primeira captura pesava $24,0 \mathrm{~g}$ e foi considerada não reprodutiva; no mês seguinte, pesava 26,5 g e dois filhotes foram encontrados mortos dentro da armadilha. Em outubro, voltou a pesar $24,0 \mathrm{~g}$ e em novembro pesava $28,5 \mathrm{~g}$ e apresentava sinais evidentes de uma nova gestação.

O tempo de permanência médio de $G$. agilis foi de $0,64 \pm$ 1,38 meses $(n=49)$, sendo de $1,0 \pm 2,29$ meses $(n=14)$ para fêmeas e de $0,51 \pm 0,82$ mês $(n=35)$ para machos. O tempo de permanência máximo registrado foi de oito meses para uma fêmea.

As capturas de G. agilis (Fig. 1) sugerem que esta espécie é menos capturada durante o período reprodutivo. Um indivíduo de G. agilis, não marcado, foi observado em atividade nas primeiras horas da manhã, deslocando-se pelo solo, para posteriormente subir em um arbusto. O comportamento mais comum durante a soltura foi o de afastamento: os animais soltos no solo subiam até uma altura de cerca de um metro para depois iniciar um deslocamento horizontal, enquanto que aqueles que foram soltos no alto, afastavam-se sem subir.

As médias das medidas tomadas de indivíduos adultos de $G$. agilis estão dentro das médias descritas para a espécie (EMmons \& Feer 1997, Eisenberg \& Redford 1999, Rossi et al. 2006), com exceção do tamanho da orelha, cuja média para machos e fêmeas é superior ao máximo descrito (Tab. III).

Micoureus demerarae foi a espécie com maior número de indivíduos capturados (240) e com maior número de eventos de captura (1287). Ocorreu nas nove grades sendo significativamente mais capturados nos landis $\left(\chi^{2}=345,98 ; \mathrm{gl}=2 ; \mathrm{p}<\right.$ $0,001)$ e na vazante $\left(\chi^{2}=81,33 ; \mathrm{gl}=2 ; \mathrm{p}<0,001 ;\right.$ Fig. 1$)$. Não houve diferença significativa no tipo de armadilha usada quando instaladas no alto, tanto para um esforço de 50 a-n $\left(\chi^{2}=\right.$ $0,51 ; \mathrm{gl}=1 ; \mathrm{p}>0,05)$ como para 100 a-n $\left(\chi^{2}=0,24 ; \mathrm{gl}=1 ; \mathrm{p}>\right.$ $0,05)$. Entretanto, armadilhas colocadas no alto resultaram em freqüência de captura significativamente maior do que as do solo ( $\left.\chi^{2}=148,40 ; \mathrm{gl}=3 ; \mathrm{p}<0,001\right)$.

O número de indivíduos descritos para cada fisionomia não está de acordo com o total de 240 devido à mudança de grade de um indivíduo: macho jovem, capturado na cordilheira-1, em maio de 2006. Foi recapturado neste habitat três vezes nos dois meses seguintes, sendo que na última dessas recapturas já era sub-adulto. No final de setembro foi recapturado, já adulto, no cambarazal-2. A distância por ele percorrida entre as grades foi de cerca de $950 \mathrm{~m}$.

A razão sexual total foi de 1,3:1 (133 machos e 106 fêmeas), variando de 1,5:1 nos cambarazais, 1,9:1 nas cordilheiras e 0,9:1 nos landis.

Dentre as fêmeas, 28 foram apenas capturadas uma vez ou recapturadas no mesmo mês da primeira captura. Desconsiderando as recapturas ocorridas em um mesmo mês e a classe etária jovem, foram feitos 341 registros de informação reprodutiva referentes a 101 fêmeas.

Micoureus demerarae também se reproduz entre o final da seca e durante a cheia, sendo o início desta estação seu pico reprodutivo (Fig. 3). Indivíduos adultos de M. demerarae foram registrados o ano todo, enquanto que a presença de jovens somente foi registrada no primeiro semestre do ano e de subadultos acompanha o padrão de freqüência relativa de sinais reprodutivos com uma defasagem de cinco meses, corroborando os registros reprodutivos (Fig. 3). Sete fêmeas foram capturadas com filhotes, sendo que uma delas em dois eventos distintos. A média de filhotes foi de 10,1 $\pm 0,8(\mathrm{n}=8)$, variando de 9 a 11 e apenas uma delas era sub-adulta. Duas fêmeas com filhotes recém-nascidos foram recapturadas no mês seguinte. Em um período de 22/23 dias seus filhotes passaram de cerca de $0,6 \mathrm{~cm}$ para aproximadamente $2,5 \mathrm{~cm}$. Uma dessas fêmeas havia perdido um filhote.

O tempo médio de permanência na grade foi de 2,36 \pm 2,73 meses $(\mathrm{n}=195)$, sendo de $2,71 \pm 2,98$ meses para fêmeas $(n=82)$ e de $2,11 \pm 2,52$ meses para machos $(n=113)$. O tempo de permanência máximo registrado foi de 15 meses, observado em uma fêmea que foi capturada e coletada no último mês de monitoramento. Esta fêmea apresentava sinais de senilidade: dentes muito desgastados, pêlos rareados e pouco vistosos no dorso e alguns pequenos tufos de pêlos brancos.

A predação foi responsável por seis das nove mortes 


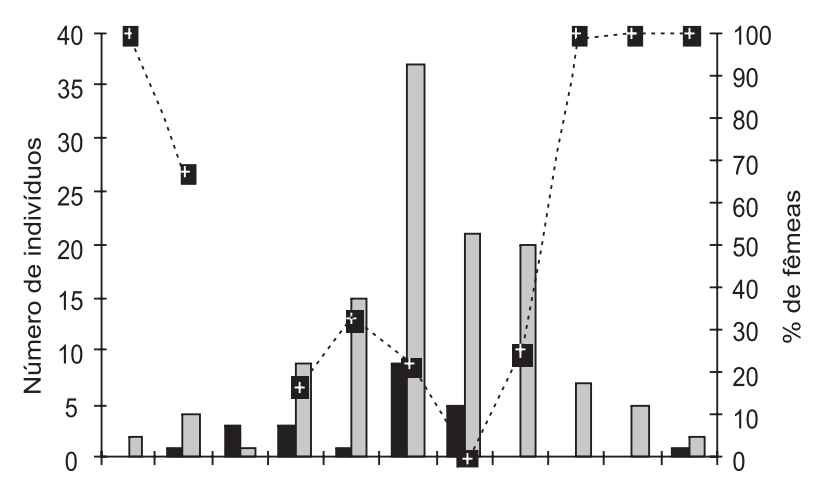

fev mar abr mai jun jul ago set out nov dez

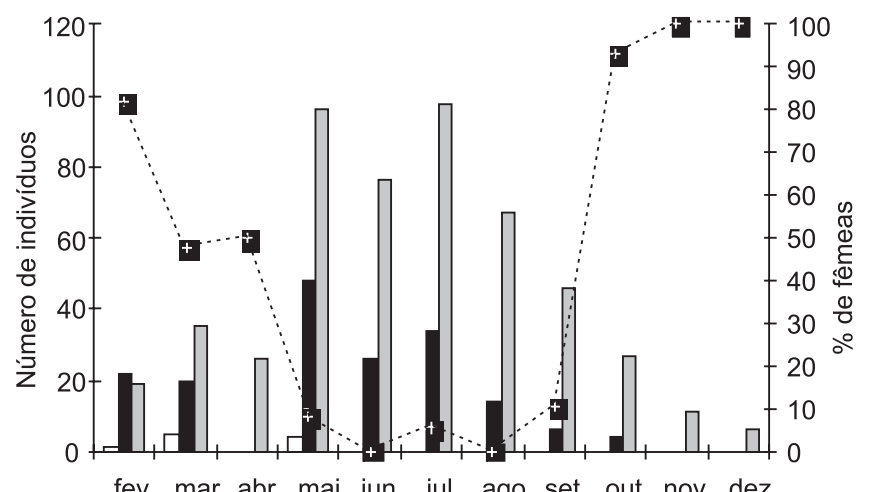

2

$\square$ jovem $\square$ sub-adulto $\square$ adulto $\cdots+\cdots$ sinais reprodutivos
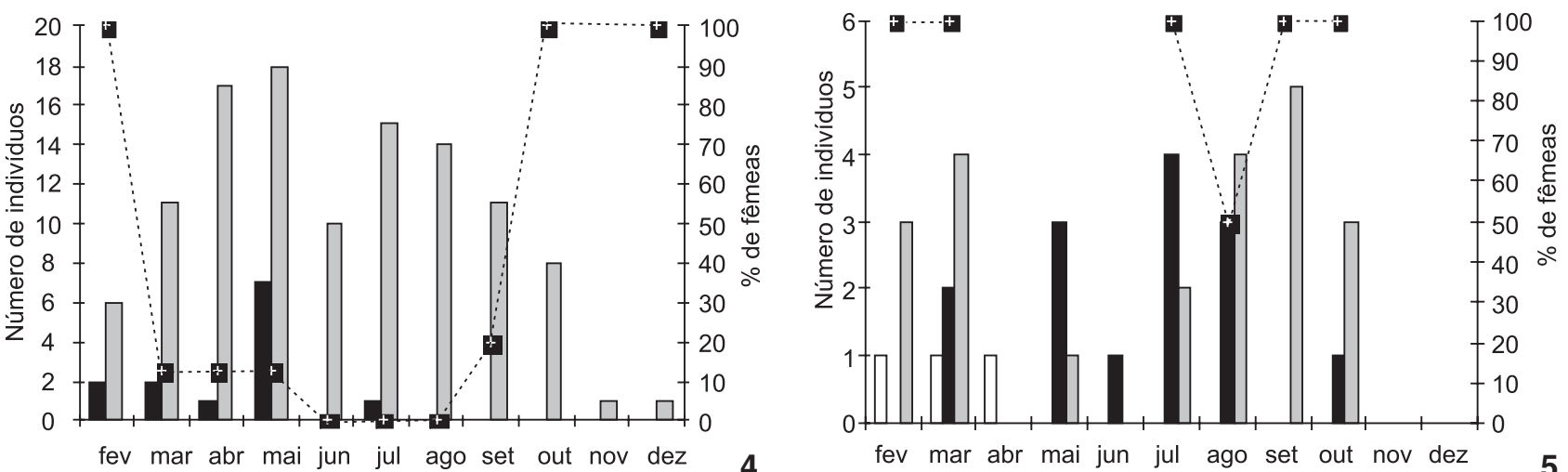

Figuras 2-5. Freqüência relativa mensal de sinais reprodutivos em fêmeas e número de indivíduos de cada classe etária ao longo do ano, a partir dos dados mensais dos anos de 2006 e 2007: (2) G. agilis; (3) M. demerarae; (4) M. domestica; (5) P. opossum.

registradas para indivíduos monitorados; outros quatro eventos deste tipo foram registrados para esta espécie, mas os restos não permitiram identificar se os indivíduos eram marcados ou não. As gaiolas colocadas no solo freqüentemente eram deslocadas do seu ponto na trilha para dentro do mato, por algum predador. Além destes episódios de predação, muitas vezes a gaiola era encontrada com o indivíduo capturado vivo, mas com algum ferimento, especialmente na cauda. Em uma ocasião, quando as armadilhas estavam sendo percorridas para a coleta das capturas daquela noite, um quati - Nasua nasua (Linnaeus, 1766) - foi observado tentando acessar um $M$. demerarae capturado em uma gaiola.

Das mortes ocorridas em shermans, pelo menos uma se deu em decorrência ao ataque de formigas Asteca sp. Estas formigas são freqüentes nos landis e a instalação das armadilhas no alto, neste habitat, evitava apoios com sua presença. Ainda assim, algumas vezes foram encontrados animais sob ataque, que eram livrados das formigas antes de serem soltos.

Em março de 2007, uma fêmea que estava sendo monitorada desde abril do ano anterior, foi capturada no landi-3, no primeiro dia de armadilhamento do mês, com nove filhotes de cerca de um mês de vida. Dois dias depois, um P. opossum foi capturado em uma sherman e dentro desta foram encontrados os restos do indivíduo adulto, oito filhotes mortos e o brinco de identificação.

Outro aspecto interessante observado em $M$. demerarae foi a mudança de coloração. Segundo Rossı et al. (2006), esta espécie apresenta “...pelagem dorsal... de coloração marromacinzentada e... ventral constituída de pêlos com base cinza e ápice creme, exceto no queixo e na região inguinal". Esta descrição é precisa, especialmente para o ventre, uma vez que se trata de uma característica diagnóstica da espécie. Entretanto, foi observado que nos primeiros cinco meses do ano a maioria dos indivíduos de $M$. demerarae apresentava uma coloração mais alaranjada, quase salmão, em toda a pelagem descrita como creme. Ao longo do ano, as recapturas dos mesmos indivíduos mostraram que a coloração retorna ao padrão creme. Este fato pode estar associado ao consumo de frutos vermelhos como os de Miconia sp. (Melastomataceae) e de Eugenia sp. (Myrtaceae), cujas sementes foram encontradas nas fezes e que são abun- 
dantes nesta época do ano (Ротт \& Pотт 1994). A mudança na coloração também foi observada em G. agilis, mas apenas em alguns indivíduos, devido à baixa captura desta espécie neste período do ano. Outros frutos consumidos por M. demerarae foram as marmeladas, Alibertia edulis (L.L. Rich.) A.C. Rich., A. sessilis (Vell.) Schum. e A. verrucosa S. Moore (Rubiaceae).

Apesar de não ter sido feito um registro sistemático do comportamento de $M$. demerarae no momento de sua soltura, observou-se que o comportamento padrão dos indivíduos era o de subir, mesmo para aqueles que, por terem sido capturados no alto, eram liberados no mesmo local. Entretanto, não subiam até o alto dossel, começando um deslocamento horizontal em uma faixa entre 2 e $4 \mathrm{~m}$. Em várias oportunidades, foi possível acompanhar este deslocamento e verificar o uso de ocos de árvores como abrigo, nos landis e cordilheiras.

Ao longo do trabalho, vários ocos foram identificados e à medida que a soltura de um indivíduo era realizada nas proximidades de um oco sabidamente utilizado por M. demerarae, especial atenção era dada para o deslocamento do indivíduo. Aparentemente, estes abrigos não têm donos, sendo utilizados por diversos indivíduos, desde que desocupados. Em uma ocasião, um indivíduo solto rumou para o abrigo em observação e encontrou-o ocupado por outro $M$. demerarae (verificado posteriormente). O comportamento agonístico observado foi a vocalização (ambos) e a abertura da boca e exibição dos dentes por parte do que estava fora do abrigo. Este embate durou cerca de um minuto, terminando com a desistência do abrigo por parte do indivíduo que havia sido solto e seu deslocamento para local fora de alcance visual. Todos os ocos registrados nos landis eram em pimenteira (Licania parvifolia Huber Chrysobalanaceae), uma das espécies de maior importância da comunidade vegetal desta fisionomia (NunEs DA CuNHA et al. 2007).

Das solturas ocorridas no solo, em algumas oportunidades foi observado comportamento agonístico com relação aos pesquisadores: ao invés de se afastarem, os indivíduos permaneceram próximos, enquanto iscávamos e instalávamos novamente a armadilha. Ficavam em pé sobre os membros posteriores, com os membros anteriores abertos, assim como a boca, balançando o corpo, lentamente, de um lado para o outro, até que fossemos embora. Em quatro ocasiões verificou-se que o indivíduo não mostrava sinais de medo ou agressividade em relação aos pesquisadores: quando soltos farejaram o saco em que carregávamos as iscas e se aproximaram. Posteriormente verificou-se tratar de animais com longo histórico de captura, que possivelmente já estavam acostumados conosco.

As médias das medidas tomadas de indivíduos adultos de $M$. demerarae estão dentro das médias descritas para a espécie (Emmons \& Feer 1997, Eisenberg \& Redford 1999, Rossi et al. 2006), mas o peso mínimo e as médias para o tamanho da orelha de machos e fêmeas no Pantanal são menores que os descritos (Tab. III).

Foram capturados 63 indivíduos de M. domestica em 150 eventos de captura. Salvo dois indivíduos capturados no landi-3 durante a seca, os demais ocorreram somente nas três cordilheiras. Não há diferença significativa na captura desta espécie entre estações nas cordilheiras $\left(\chi^{2}=0,26 ; \mathrm{gl}=2 ; \mathrm{p}>0,05 ;\right.$ Fig. 1$)$. São significativamente mais capturados no solo $\left(\chi^{2}=107,34 ; \mathrm{gl}=3\right.$; $\mathrm{p}<0,001)$, mas não há diferença significativa no tipo de armadilha usada neste estrato $\left(\chi^{2}=1,66 ; \mathrm{gl}=1 ; \mathrm{p}>0,05\right)$.

A razão sexual total foi de 1,2:1 (34 machos e 29 fêmeas), variando de 0,8 a 2:1 entre as cordilheiras. Apenas um registro da classe etária sub-adulto foi feito, os demais eram adultos.

Desconsiderando as recapturas ocorridas em um mesmo mês, foram feitos 56 registros de informação reprodutiva referentes a 28 fêmeas. Não foi registrada atividade reprodutiva em $M$. domestica no período entre o final da vazante e o início da seca (Fig. 4). Cinco fêmeas foram capturadas com filhotes, sendo a média de filhotes de 9,6 $\pm 1,7$, variando de 7 a 11 .

Mais da metade dos indivíduos marcados (51,6\%) foi capturada somente uma vez ou foi recapturada no mesmo mês da primeira captura. $\mathrm{O}$ tempo de permanência médio foi de 1,48 \pm 2,27 meses $(\mathrm{n}=50)$, sendo de $1,65 \pm 2,48$ meses para fêmeas $(\mathrm{n}=23)$ e de $1,33 \pm 2,11$ para machos $(\mathrm{n}=27)$. A permanência máxima registrada foi de sete meses, observada em duas fêmeas e dois machos. As médias das medidas tomadas de indivíduos adultos de $M$. domestica estão dentro das médias descritas para a espécie (Emmons \& Feer 1997, Eisenberg \& Redford 1999, Rossi et al. 2006), registrando apenas tamanhos mínimos do comprimento cabeça e corpo menores (Tab. III).

Foram capturados 27 indivíduos de $P$. opossum em 47 eventos de captura. Ocorreu nas três réplicas de cada um dos habitats alagáveis, sendo significativamente mais capturados nos cambarazais $\left(\chi^{2}=7,68 ; \mathrm{gl}=1 ; \mathrm{p}<0,05\right)$ e durante a cheia $\left(\chi^{2}=10,69 ; \mathrm{gl}=2 ; \mathrm{p}<0,01 ;\right.$ Fig. 1$)$. Não há diferença significativa no uso de armadilhas instaladas no alto e no solo $\left(\chi^{2}=\right.$ $0,53 ; \mathrm{gl}=1 ; \mathrm{p}>0,05)$, sendo que o uso de shermans para a captura desta espécie é limitado pelo tamanho do indivíduo, capturando somente $16,1 \%$ dos indivíduos adultos.

A razão sexual total foi de 1,1:1 (14 machos e 13 fêmeas), sendo de 1:1 nos cambarazais e de 1,7:1 nos landis. Desconsiderando as recapturas ocorridas em um mesmo mês e a classe etária jovem, foram feitos 17 registros de informação reprodutiva referentes a 12 fêmeas. A lacuna de registros reprodutivos entre os meses de abril e julho e entre novembro e janeiro não permite uma conclusão sobre período reprodutivo de $P$. opossum, mas a presença de jovens e sub-adultos ao longo do ano sugerem que a reprodução desta espécie pode ser contínua (Fig. 5). Nos sete meses em que fêmeas adultas foram capturadas, pelo menos uma estava com filhotes no marsúpio. Sete fêmeas foram capturadas com filhotes, uma delas em dois eventos distintos (fevereiro e agosto de 2006). O número de filhotes não pode ser precisamente avaliado, devido ao marsúpio e, em alguns casos, o tamanho dos filhotes; as ninhadas variaram de pelo menos dois a pelo menos cinco. Uma destas capturas chamou a atenção pelo tamanho dos filhotes no marsúpio: apresentavam pêlos e unhas bastante desenvolvidas e já não cabi- 
am mais totalmente dentro do marsúpio, com pernas e caudas saindo fora deste. Quando solta a mãe apresentou enorme dificuldade na locomoção e na manutenção do equilíbrio ao caminhar por uma rama de "cipó-de-arraia" (Cissus spinosa Camb. - Vitaceae).

Três indivíduos foram encontrados mortos entre setembro e outubro, possivelmente devido à desidratação. Um macho adulto apresentou sinais clínicos de desidratação como a diminuição da mobilidade da pele, verificado através de seu pinçamento, olhos embaçados e apatia, não estando reativo a estímulos e deixando-se manusear. Após oferta de água, foi deixado em observação e cerca de 20 minutos depois o animal apresentou sinais de recuperação, com maior mobilidade e brilho nos olhos. Como sua gaiola estava aberta, lentamente foi se afastando, deslocando-se pelo solo.

Treze indivíduos (65\%) foram capturados apenas uma vez ou recapturados somente no mesmo mês da primeira captura.
O tempo de permanência médio foi de $0,95 \pm 1,61$ mês $(\mathrm{n}=$ 20 ), sendo de $1,33 \pm 1,94$ mês para fêmeas $(n=9)$ e de $0,64 \pm$ 1,29 mês para machos $(\mathrm{n}=11)$. A permanência máxima foi de cinco meses, observada em uma fêmea.

O comportamento de $P$. opossum quando liberado era o de afastamento, mas mantendo-se no mesmo estrato em que foi capturado e solto (solo ou alto). Das 13 capturas efetuadas no solo, quatro indivíduos $(30,7 \%)$ abrigaram-se em buracos no solo. Além da predação sobre $M$. demerarae, foram observados restos de caranguejo nas fezes de P. opossum. Este Trichodactylidae, provavelmente Dilocarcinus pagei Stimpson, 1861 (Célio Magalhães, com. pes.), é muito abundante nos habitats alagáveis onde $P$. opossum foi capturado e sua coloração vermelha confere bastante visibilidade aos indivíduos. As médias das medidas tomadas de indivíduos adultos de $P$. opossum estão dentro das médias descritas para a espécie (EMmons \& FEeR 1997, Eisenberg \& Redford 1999, Rossi et al. 2006) (Tab. III).

\begin{tabular}{|c|c|c|c|c|c|c|c|}
\hline Espécies & Cabeça & Cabeça e corpo & Cauda & Orelha & PPD com unha & PPD sem unha & Peso \\
\hline \multicolumn{8}{|l|}{ Gracilinanus agilis } \\
\hline \multirow[t]{2}{*}{ Fêmea (min-máx) } & $\begin{array}{c}28,9 \pm 1,5 \\
(26,5-31,3)\end{array}$ & $\begin{array}{c}96,0 \pm 7,3 \\
(83,5-109,0)\end{array}$ & $\begin{array}{c}126,9 \pm 9,2 \\
(113,0-138,0)\end{array}$ & $\begin{array}{c}20,9 \pm 0,8 \\
(20,0-22,0)\end{array}$ & $\begin{array}{c}15,3 \pm 0,7 \\
(13,9-16,3)\end{array}$ & - & $\begin{array}{c}17,8 \pm 3,6 \\
(12,5-25,0)\end{array}$ \\
\hline & $(n=11)$ & $(n=11)$ & $(n=11)$ & $(n=11)$ & $(n=10)$ & & $(n=20)$ \\
\hline \multirow[t]{2}{*}{ Macho (min-máx) } & $\begin{array}{c}31,1 \pm 2,0 \\
(23,0-34,7)\end{array}$ & $\begin{array}{c}107,9 \pm 9,9 \\
(82,0-123,0)\end{array}$ & $\begin{array}{c}140,6 \pm 9,5 \\
(113,0-157,0)\end{array}$ & $\begin{array}{c}22,7 \pm 1,7 \\
(18,0-29,0)\end{array}$ & $\begin{array}{c}16,4 \pm 0,9 \\
(14,0-17,9)\end{array}$ & - & $\begin{array}{c}27,8 \pm 5,6 \\
(15,0-43,0)\end{array}$ \\
\hline & $(n=43)$ & $(n=42)$ & $(n=43)$ & $(n=43)$ & $(n=40)$ & & $(n=51)$ \\
\hline \multicolumn{8}{|l|}{ Micoureus demerarae } \\
\hline \multirow[t]{2}{*}{ Fêmea (min-máx) } & $\begin{array}{c}43,1 \pm 2,9 \\
(38,1-53,0)\end{array}$ & $\begin{array}{c}150,8 \pm 15,7 \\
(123,0-186,0)\end{array}$ & $\begin{array}{c}201,8 \pm 15,4 \\
(177,0-237,0)\end{array}$ & $\begin{array}{c}28,4 \pm 2,4 \\
(23,0-32,5)\end{array}$ & $\begin{array}{c}23,4 \pm 1,5 \\
(20,0-28,0)\end{array}$ & $\begin{array}{c}21,9 \pm 1,5 \\
(18,0-26,5)\end{array}$ & $\begin{array}{c}63,6 \pm 17,0 \\
(36,0-145,0)\end{array}$ \\
\hline & $(n=36)$ & $(n=35)$ & $(n=35)$ & $(n=36)$ & $(n=34)$ & $(n=34)$ & $(n=168)$ \\
\hline \multirow[t]{2}{*}{ Macho (min-máx) } & $\begin{array}{c}45,8 \pm 3,1 \\
(39,7-53,2)\end{array}$ & $\begin{array}{c}166,1 \pm 16,8 \\
(119,0-201,0)\end{array}$ & $\begin{array}{c}223,8 \pm 14,8 \\
(191,5-257,0)\end{array}$ & $\begin{array}{c}30,6 \pm 2,2 \\
(22,0-34,0)\end{array}$ & $\begin{array}{c}25,9 \pm 1,7 \\
(22,1-29,7)\end{array}$ & $\begin{array}{c}24,0 \pm 1,6 \\
(21,0-27,8)\end{array}$ & $\begin{array}{c}95,1 \pm 25,1 \\
(48,5-165,5)\end{array}$ \\
\hline & $(n=78)$ & $(n=76)$ & $(n=78)$ & $(n=78)$ & $(n=69)$ & $(n=69)$ & $(n=274)$ \\
\hline \multicolumn{8}{|l|}{ Monodelphis domestica } \\
\hline \multirow[t]{2}{*}{ Fêmea (min-máx) } & $\begin{array}{c}37,3 \pm 2,5 \\
(31,5-42,0)\end{array}$ & $\begin{array}{c}124,5 \pm 8,7 \\
(113,0-142,0)\end{array}$ & $\begin{array}{c}71,7 \pm 4,0 \\
(64,0-77,5)\end{array}$ & $\begin{array}{c}21,9 \pm 1,5 \\
(18,0-24,0)\end{array}$ & $\begin{array}{c}18,1 \pm 1,0 \\
(15,5-19,5)\end{array}$ & $\begin{array}{c}16,7 \pm 0,7 \\
(15,8-18,0)\end{array}$ & $\begin{array}{c}40,5 \pm 6,2 \\
(32,0-67,5)\end{array}$ \\
\hline & $(n=18)$ & $(n=18)$ & $(n=18)$ & $(n=18)$ & $(n=18)$ & $(n=17)$ & $(n=33)$ \\
\hline \multirow[t]{2}{*}{ Macho (min-máx) } & $\begin{array}{c}54,6 \pm 2,4 \\
(34,0-44,5)\end{array}$ & $\begin{array}{c}141,0 \pm 15,1 \\
(114,0-164,0)\end{array}$ & $\begin{array}{c}79,6 \pm 6,3 \\
(68,0-91,5)\end{array}$ & $\begin{array}{c}23,3 \pm 2,4 \\
(17,5-29,5)\end{array}$ & $\begin{array}{c}19,1 \pm 1,3 \\
(15,0-22,0)\end{array}$ & $\begin{array}{c}17,6 \pm 1,3 \\
(14,0-20,5)\end{array}$ & $\begin{array}{l}57,9 \pm 12,9 \\
(35,0-89,5)\end{array}$ \\
\hline & $(\mathrm{n}=29)$ & $(n=29)$ & $(\mathrm{n}=29)$ & $(n=29)$ & $(n=29)$ & $(n=29)$ & $(n=59)$ \\
\hline \multicolumn{8}{|l|}{ Philander opossum } \\
\hline \multirow[t]{2}{*}{ Fêmea (min-máx) } & $\begin{array}{c}67,1 \pm 4,1 \\
(62,2-73,6)\end{array}$ & $\begin{array}{c}243,4 \pm 10,4 \\
(228,0-256,0)\end{array}$ & $\begin{array}{c}268,1 \pm 12,8 \\
(246,0-286,0)\end{array}$ & $\begin{array}{c}33,3 \pm 5,1 \\
(25,0-38,0)\end{array}$ & $\begin{array}{c}35,9 \pm 1,7 \\
(34,1-39,2)\end{array}$ & $\begin{array}{c}33,9 \pm 1,9 \\
(31,7-37,8)\end{array}$ & $\begin{array}{c}198,3 \pm 53,5 \\
(165,0-260,0)\end{array}$ \\
\hline & $(n=7)$ & $(n=7)$ & $(n=7)$ & $(n=7)$ & $(n=7)$ & $(n=7)$ & $(n=3)$ \\
\hline \multirow[t]{2}{*}{ Macho (min-máx) } & $\begin{array}{c}74,0 \pm 1,6 \\
(71,4-75,6)\end{array}$ & $\begin{array}{c}271,2 \pm 7,9 \\
(258,0-279,0)\end{array}$ & $\begin{array}{c}292,0 \pm 5,9 \\
(286,0-299,0)\end{array}$ & $\begin{array}{c}38,3 \pm 1,3 \\
(36,5-40,0)\end{array}$ & $\begin{array}{c}39,0 \pm 2,8 \\
(35,3-42,6)\end{array}$ & $\begin{array}{c}37,5 \pm 3,5 \\
(32,5-42,0)\end{array}$ & $\begin{array}{c}344,6 \pm 55,0 \\
(293,0-450,0)\end{array}$ \\
\hline & $(n=5)$ & $(n=5)$ & $(n=5)$ & $(n=5)$ & $(n=5)$ & $(n=5)$ & $(n=8)$ \\
\hline
\end{tabular}




\section{DISCUSSÃO}

O sucesso total de captura obtido para os habitats florestados amostrados no Pantanal (6,3\%) foi maior que os encontrados no Cerrado, no Pantanal da Nhecolândia e em áreas de Mata Atlântica mais distantes da costa (Tab. IV). A porcentagem de capturas de marsupiais com relação aos roedores também se destaca por assemelhar-se mais aos estudos realizados em Mata Atlântica do que em áreas de Cerrado e Pantanal.

Apenas G. agilis e M. demerarae foram capturados nas três fisionomias estudadas e podem ser considerados generalistas de habitat. $P$. opossum está restrita a áreas alagáveis, enquanto $M$. domestica pode ser considerada praticamente exclusiva de cordilheiras (Tab. II). Embora C. philander e D. albiventris somente terem sido capturadas em landis não se pode afirmar que sejam exclusivas deste habitat.

Apesar de ser eventualmente capturada no solo (FonseCA \& KierulfF 1989, Stallings 1989), C. philander é descrita como sendo arborícola de médio a alto dossel (Passamani 1995, Leite et al. 1996, Vieira \& Monteiro-Filho 2003), sugerindo que a altura de instalação das armadilhas não acessa plenamente o nicho espacial desta espécie. Entretanto, cabe notar que a altura de instalação das armadilhas que capturaram C. philander variou entre $1 / 5$ a $1 / 2$ em relação à altura do dossel do ponto e imediações, mostrando uma amplitude de uso espacial bem maior. Assim, C. philander pode ter sido sub-amostrado, em função da altura de instalação das armadilhas, possivelmente ocorrendo em outros habitats no Pantanal, já que a espécie é descrita para vários tipos de florestas, além de jardins e plantações (EMmons \& FeER 1997).

Didelphis albiventris chama a atenção por ter tido apenas um indivíduo capturado após onze meses de coleta sistemática. Onde esta espécie ocorre, é descrita como bastante comum, sendo freqüentemente a espécie mais capturada, ocorrendo tanto em habitats florestados como em áreas abertas (FonsecA \& Redford 1984, Alho et al. 1986, Marinho-Filho et al. 2002, Rodrigues et al. 2002). Marinho-Filho \& Guimarães (2001) mostram que esta espécie ocorre em matas ribeirinhas em sete das oito áreas estudadas no Distrito Federal. Rodrigues et al. (2005) apontam a presença de $D$. albiventris tanto para a planície pantaneira como para seus arredores. Entretanto, LACHER \& ALHO (1989) não a capturaram no Pantanal da Nhecolândia. Relatos de moradores locais afirmam que $D$. albiventris é freqüentemente visto próximos às suas casas, em cozinhas, depósitos de rações e em locais de descarte de lixo. Espécies de Didelphis Linnaeus, 1758 são generalistas de habitat e dieta, que se adaptam muito bem a áreas fragmentadas, onde a fauna de carnívoros esteja depauperada ou eliminada. Nestas situações, as espécies de Didelphis tendem a se tornar dominantes, conforme sugerido por Fonseca (1989) e Fonseca \& Robinson (1990). Em habitats pouco alterados, onde a comunidade de mamíferos de médio e maior porte e, especialmente, os predadores de topo ainda ocorrem, como é o caso da região estudada, Didelphis tende a ser muito menos abundante.

O padrão de atividade reprodutiva de G. agilis descrito para o Pantanal também foi encontrado por MAREs \& ERNEST (1995) para população estudada em mata de galeria. Monodelphis Burnett, 1830 é um dos poucos gêneros, dentre os marsupiais brasileiros, de hábito terrestre (FonsECA et al. 1996). Entretanto, $8,1 \%$ das capturas de $M$. domestica no Pantanal ocorreram em armadilhas instaladas no alto. AlHo et al. (1986) também observaram a capacidade desta espécie em subir, entretanto, associaram esta espécie a mata de galeria em detrimento de habitats mais secos como cerrado e campo. Apesar de M. domestica ser descrita como uma espécie que se reproduz ao longo de todo o ano (STREILEIN 1982a), nossos resultados sugerem que não há atividade reprodutiva no período entre o final da vazante e o início da seca no Pantanal. Este padrão também foi encontrado por Bergallo \& Cerqueira (1994), que descrevem a estação reprodutiva coincidente com a estação chuvosa e associada ao fotoperiodismo.

O tempo de permanência médio de fêmeas é maior que o de machos para todas as espécies, variando de 0,51 mês para machos de G. agilis a 2,71 meses para fêmeas de $M$. demerarae. $\mathrm{O}$ desaparecimento dos indivíduos pode ser atribuído à migração, à predação e ao tamanho da área de vida. A migração ou dispersão foi observada em um macho de M. demerarae e a ausência de G. agilis nas cordilheiras durante a cheia de 2007 sugere uma migração local, já que a espécie foi observada antes e depois deste evento neste habitat.

Tabela IV. Número de espécies de marsupiais ( $\mathrm{N}^{\circ} \mathrm{sp} . \mathrm{M}$ ) e de roedores ( $\left.\mathrm{N}^{\circ} \mathrm{sp} . \mathrm{R}\right)$, porcentagem (\% capt.), sucesso e esforço de captura (armadilhas-noite) de diversos estudos em biomas brasileiros.

\begin{tabular}{|c|c|c|c|c|c|c|c|}
\hline \multirow{2}{*}{$\begin{array}{l}\text { Estudo } \\
\text { FonsECA \& REDFORD (1984) }\end{array}$} & \multirow{2}{*}{$\begin{array}{l}\text { Bioma/habitats } \\
\text { Cerrado/mata de galeria }\end{array}$} & \multicolumn{2}{|c|}{$\begin{array}{c}\mathrm{N}^{\circ} \text { sp. M } \\
\text { (\% capturas) }\end{array}$} & \multicolumn{2}{|c|}{$\begin{array}{c}\mathrm{N}^{\circ} \text { sp. } \mathrm{R} \\
\text { (\% capturas) }\end{array}$} & \multirow{2}{*}{$\begin{array}{c}\text { Sucesso } \\
(\%) \\
2,4\end{array}$} & \multirow{2}{*}{$\begin{array}{c}\begin{array}{c}\text { Esforço } \\
(a-n)\end{array} \\
2584\end{array}$} \\
\hline & & 2 & $(-)$ & 6 & $(-)$ & & \\
\hline FonsECA \& KIERULFF (1989) & Mata Atlântica/floresta & 8 & $(69,4)$ & 12 & $(30,6)$ & 2,4 & 57120 \\
\hline Lacher \& Alho (1989) & Pantanal/campo, cerrado, transição e floresta & 1 & $(1,3)$ & 6 & $(98,7)$ & 4,2 & 3582 \\
\hline StALLINGS (1989) & Mata Atlântica/floresta, campo úmido & 6 & $(55,3)$ & 11 & $(44,7)$ & 3,3 & 32940 \\
\hline RODRIGUes et al. (2002) & Cerrado/mata de galeria, cerrado, vereda, campo & 10 & $(14,0)$ & 12 & $(86,0)$ & 4,2 & 13562 \\
\hline Este estudo (2007) & Pantanal/florestas & 6 & $(70,1)$ & 8 & $(29,9)$ & 6,3 & 38635 \\
\hline
\end{tabular}


A relação entre o tamanho da área amostrada e o tamanho da área de vida também podem conduzir a uma situação de desaparecimento dos indivíduos quanto maior esta for em relação àquela e o quão distante da região central da área de vida estiver a área amostrada. Moraes \& Chiarello (2005) mostram, através de rádio-telemetria, que a área de uso de $M$. paraguayanus (Rossi et al. 2006) é maior (1,3-5,9 ha para fêmeas e 4,0-10,9 ha para machos) do que as áreas amostradas, cuja média é de 1,92 $\pm 0,34$ ha. A predação e tentativas de predação foram observadas diversas vezes para três das seis espécies estudadas, sendo que uma delas (P. opossum) atuou como predador. Esta interação tem sido mostrada como importante força na estruturação de comunidades (BEGON et al. 1996) e um tempo de permanência individual baixo em cada localidade pode ser um reflexo disto.

O período reprodutivo dos marsupiais estudados, salvo D. albiventris e C. philander cujas informações são mais escassas, está concentrado no período de cheia, com início no final da estação seca. Este resultado está de acordo com CERQUeIrA (2005) que aponta o aumento da duração do dia como fator próximo para o desencadeamento da estação reprodutiva. Além disso, Cerqueira (2005) mostra que a duração da estação reprodutiva está correlacionada com a latitude, podendo ser contínua perto do equador, reduzindo-se em latitudes maiores. Esta correlação também age sobre o número e tamanho das ninhadas, sendo aquele diminuído e este aumentado com a redução da duração da estação reprodutiva (CERQUEIRA 2005).

Patton et al. (2000) descrevem uma ninhada de sete filhotes para $M$. demerarae e sugerem que a reprodução desta espécie ocorra o ano todo, conforme suas observações realizadas no Rio Juruá, entre $2^{\circ}$ e $10^{\circ}$ de latitude Sul. Comparando estes dados com os registrados no Pantanal, M. demerarae corrobora a hipótese de CERQUeIRA (2005), com a redução do período reprodutivo e o aumento do número médio de filhotes em latitudes maiores. Do mesmo modo, comparando aos dados de M. domestica descritos por Bergallo \& Cerqueira (1994), registrou-se no Pantanal um número médio de filhotes maior para uma latitude maior, apesar da antecipação da estação reprodutiva. Apesar de CERQUEIRA (2005) ter desenvolvido as correlações dos fatores ambientais com a reprodução de marsupiais para um enorme conjunto de dados oriundos do leste do Brasil, estas correlações também podem ser válidas para o Pantanal e Amazônia (Monterro-Filho \& CÁCERES 2006), uma vez que o eixo de ação é latitudinal.

Por outro lado, FleCK \& HaRder (1995), em estudo na floresta amazônica, concluíram que a atividade reprodutiva de marsupiais estava positivamente relacionada com as chuvas e a produção de frutos. O'ConNell (1989) também registra o período reprodutivo de marsupiais na estação chuvosa. STREilein (1982b), em uma revisão sobre o comportamento, ecologia e distribuição de marsupiais sul-americanos, afirma que algumas espécies de marsupiais com ampla distribuição geográfica provavelmente apresentam estratégias reprodutivas distintas para diferentes regiões.
Stallings (1989) discute que altas densidades locais de marsupiais podem ser encontradas em áreas onde haja abundância de recurso alimentar. No caso do Pantanal, esta hipótese pode ser fortalecida pelo fato das áreas florestadas representarem "ilhas" em uma matriz de campo, oferecendo concentração de recursos e, em maior ou menor grau, todas as espécies aqui descritas incluem frutos em suas dietas (FonsECa et al. 1996, Marinho-Filho \& Guimarães 2001). Durante a cheia, as florestas inundáveis estão frutificando (NunEs DA CUNHA \& JuNK 1996), enquanto as cordilheiras não sofrem alagamento. Durante a seca, muitas espécies de cordilheira estão produzindo sementes oleaginosas (NuNES DA CUNHA \& JUNK 1996), enquanto os landis e cambarazais podem oferecer maior sombreamento e conforto térmico. Apesar de landis e cambarazais serem florestas inundáveis, a diversidade florística dos cambarazais é reduzida, por serem estandes monotípicos com dominância de $V$. divergens. Este fato pode justificar a menor riqueza de marsupiais observada em relação aos landis. Por fim, os habitats florestados oferecem maior proteção quanto a predação do que áreas abertas de campos, mesmo para animais noturnos, como os marsupiais.

\section{AGRADECIMENTOS}

Os autores agradecem a Arlindo V. de Lima, pela ajuda nos trabalhos de campo, Antônio C.C. Maciel e Nilton V. de Pinho, proprietários das fazendas Aparecida e Retiro Novo, pela permissão da realização desta pesquisa em suas propriedades. Ao Centro de Pesquisas do Pantanal, pelo apoio financeiro; ao CNPq, pela bolsa de produtividade científica concedida a JMF. Ao Programa de Pós-graduação em Biologia Animal da Universidade de Brasília e CNPq pela bolsa de doutorado concedida a MA. Esta é uma contribuição do Núcleo de Estudos Ecológicos do Pantanal (NEPA/UFMT).

\section{LITERATURA CITADA}

Alho, C.J.R.; L.A. Pereira \& A.C. Paula. 1986. Patterns of habitat utilization by small mammals populations in cerrado biome of central Brazil. Mammalia 50 (4): 447-460.

Arieira, J. \& C. Nunes da Cunha. 2006. Fitossociologia de uma floresta inundável monodominante de Vochysia divergens Pohl. (Vochysiaceae), no Pantanal Norte, MT, Brasil. Acta Botânica Brasileira 20 (3): 569-580.

Begon, M.; J.L. HARPER \& C.R. TownSEND. 1996. Ecology: individuals, populations and communities. London, Blackwell Science, 1068p.

Bergallo, H.G. \& R. Cerqueira. 1994. Reproduction and growth of the opossum Monodelphis domestica (Mammalia: Didelphidae) in northeastern Brazil. Journal of Zoology (Lond.) 232: 551-563.

BRASIL 1997. Plano de Conservação da Bacia do Alto Paraguai (PCBAP) - Diagnóstico dos Meios Físico e Biótico (Meio Biótico). Brasília, Ministério do Meio Ambiente, vol. 1, 369p. 
Cerqueira, R. 2005. Fatores ambientais e a reprodução de marsupiais e roedores no leste do Brasil. Arquivos do Museu Nacional 63 (1): 29-39.

da Silva, C.J.; K.M. Wantzen; C. Nunes da Cunha \& F.A. Machado. 2001. Biodiversity in the Pantanal wetland, Brazil, p. 187215. In: B. Gopal; W.J. Junk \& J.A. Davis (Eds). Biodiversity in wetlands: assessment, function and conservation. Leiden, Backhuys Publ., vol. 2, 317p.

EISENBERG, J.F \& K.H. REDFORD. 1999. Mammals of the Neotropics: the Central Neotropics (Ecuador, Peru, Bolivia, Brazil). Chicago, University of Chicago Press, 609p.

EMmONs, L.H. \& F. FEER. 1997. Neotropical rainforest mammals: a field guide. Chicago, University of Chicago Press, 307p.

Fleck, D.W. \& J.D. Harder. 1995. Ecology of marsupials in two Amazonian rain forests in northeastern Peru. Journal of Mammalogy 76 (3): 809-818.

FONSECA, G.A.B. 1989. Small mammal species diversity in Brazilian tropical primary and secondary forests of different sizes. Revista Brasileira de Zoologia 6 (3): 381-422.

FonseCA, G.A.B. \& M.C.M. KierulfF. 1989. Biology and natural history of brazilian Atlantic Forest small mammals. Bulletin of Florida State Museum, Biological Science, 34 (4): 99-152.

FonsecA, G.A.B. \& J.G. Robinson. 1990. Forest size and structure: competitive and predatory effects on small mammal communities. Biological Conservation 53: 265-294.

Fonseca, G.A.B. \& K.H. RedFord. 1984. The mammals of IBGE's Ecological Reserve, Brasília, and an analysis of the role of gallery forests in increasing diversity. Revista Brasileira de Biologia 44 (4): 517-523.

Fonseca, G.A.B.; G. Herrmann; Y.L.R. Leite; R.A. Mittermeier; A.B. Rylands \& J.L. PatTon. 1996. Lista anotada dos mamíferos do Brasil. Conservation International, Occasional Papers. Conservation Biology 4: 1-38.

Junk, W.J. \& C.J. Da Silva. 1999. O conceito do pulso de inundação e suas implicações para o Pantanal Mato Grossense, p. 17-28. In: Anais do II Simpósio sobre Recursos Naturais e Sócio-econômicos do Pantanal - Manejo e Conservação. Corumbá, EMBRAPA Pantanal, 535p.

LACHER, T.E. \& C.J.R. AlHo. 1989. Microhabitat use among small mammals in the Brazilian Pantanal. Journal of Mammalogy 70 (2): 396-401.

Leite, Y.L.R.; L.P. Costa \& J.R. Stallings. 1996. Diet and vertical space use of three sympatric opossums in a Brazilian Atlantic Forest reserve. Journal of Tropical Ecology 12: 435-440.

Macedo, J.; D. Loretto; M.V. Vieira \& R. Cerqueira. 2006. Classes de desenvolvimento em marsupiais: um método para animais vivos. Mastozoologia Neotropical 13 (1): 133-136.

Mares, M.A. \& K.A. ERnest. 1995. Population and community ecology of small mammals in a gallery forest of central Brazil. Journal of Mammalogy 76 (3): 750-768.

Mares, M.A.; J.K. Braun \& D. Gettinger. 1989. Observations on the distribution and ecology of the small mammals of the cerrado grasslands of central Brazil. Annals of Carnegie
Museum 58: 1-60.

Marinho-Filho, J. \& M.M. Guimarães. 2001. Mamíferos das matas de galeria e das matas ciliares do Distrito Federal, p. 529557. In: J.F. Ribeiro; J.C. Silva \& C.E.L. FonseCa (Eds). Cerrado: caracterização e recuperação de matas de galeria. Planaltina, Embrapa Cerrados, 899p.

Marinho-Filho, J.; F.H.G. Rodrigues \& K.M. Juarez. 2002. The Cerrado mammals: diversity, ecology, and natural history, p. 266-284. In: P.S. Oliveira \& R.J. Marquis (Eds). The Cerrados of Brazil: ecology and and natural history of a Neotropical Savanna. New York, Columbia University Press, $424 \mathrm{p}$.

Monteiro-Filho, E.L.A. \& N.C. CÁceres. 2006. Biologia Reprodutiva de fêmeas de marsupiais didelfídeos, p 99-110. In: N.C. Cáceres \& E.L.A. Monteiro-Filho (Eds). Os marsupiais do Brasil - biologia, ecologia e evolução. Campo Grande, Editora UFMS, 364p.

Moraes Jr, E.A. \& A.G. Chiarello. 2005. A radio tracking study of home range and movements of the marsupial Micoureus demerarae (Thomas) (Mammalia, Didelphidae) in the Atlantic forest of south-eastern Brazil. Revista Brasileira de Zoologia 22 (1): 85-91.

Nunes da Cunha, C. \& W.J. Junk. 1996. Composição florística de capões e cordilheiras: localização das espécies lenhosas quanto ao gradiente de inundação no Pantanal de Poconé, MT Brasil, p. 387-405. In: Anais do II Simpósio sobre Recursos Naturais e Sócio-econômicos do Pantanal. Manejo e Conservação. Corumbá, EMBRAPA Pantanal, 535p.

Nunes da Cunha, C. \& W.J. Junk. 2004. Year-to-year changes in water level drive the invasion of Vochysia divergens in Pantanal grasslands. Applied Vegetation Science 7: 103-110.

Nunes da Cunha, C.; P. Rawiel; K.M. Wantzen; W.J. Junk \& A. Lemes do PRADO. 2006. Mapping and characterization of vegetation units by means of Landsat imagery and management recommendations for the Pantanal of Mato Grosso (Brazil), North of Poconé. Amazoniana 19: 1-32.

Nunes da Cunha, C.; W.J. Junk \& H.F. Leitão-Filho. 2007. Woody vegetation in the Pantanal of Mato Grosso, Brazil: a preliminary typology. Amazoniana 19: 159-184.

O'Connell, M.A. 1989. Population dynamics of neotropical small mammals in seasonal habitats. Journal of Mammalogy 70 (3): 532-548.

PASSAMANI, M. 1995. Vertical stratification of small mammals in Atlantic forest. Mammalia 59 (2): 276-279.

Patton, J.L.; M.N.F. da Silva \& J.R. Malcolm. 2000. Mammals of the Rio Juruá and the evolutionary and ecological diversification of Amazonia. Bulletin of the American Museum of Natural History 244: 1-306.

Ротт, A. \& V.J. Ротт. 1994. Plantas do Pantanal. Corumbá, Embrapa, 320p.

Prance, G.T. \& G.B. Schaller. 1982. Preliminary study of some vegetation types of the Pantanal, Mato Grosso, Brazil. Brittonia 34 (2): 228-251. 
Reis, N.R.; A.L. Peracchi; W.A. Pedro \& I.P. Lima. 2006. Mamíferos do Brasil. Londrina, Edifurb, 437p.

Rodrigues, F.H.G.; L. Silveira; A.T.A. Jácomo; A.P. Carmignotto; A.M.R. Bezerra; D.C. Coelho; H. Garbogini; J. Pagnozzi \& A. HAss. 2002. Composição e caracterização da fauna de mamíferos do Parque Nacional das Emas, Goiás, Brasil. Revista Brasileira de Zoologia 19 (2): 589-600.

Rodrigues, F.H.G.; I.M. Medri; W.M. Tomas \& G.M. Mourão. 2005. Revisão do conhecimento sobre a ocorrência e distribuição de mamíferos no Pantanal. Corumbá, EMBRAPA Pantanal, Série Documentos, 38, 41p.

Rossi, R.V.; G.V. Bianconi \& W.A. Pedro. 2006. Ordem Didelphimorphia, p. 27-66. In: N.R. Reis; A.L. Peracchi; W.A. Pedro \& I.P. Lima (Eds). Mamíferos do Brasil. Londrina, Edifurb, $437 \mathrm{p}$.

Stallings, J.R. 1989. Small mammal inventories in an Eastern Brazilian Park. Bulletin of Florida State Museum, Biolo- gical Science 34 (4): 153-200.

Streilein, K.E. 1982a. The ecology of small mammals in the semiarid brazilian caatinga. III. Reproductive biology and population ecology. Annals of Carnegie Museum 51: 251269.

Streilein, K.E. 1982b. Behavior, ecology, and distribution of South American marsupials. Special Publication Pymatuning Laboratory of Ecology 6: 231-250.

Vieira, E.M. \& E.L.A. Monteiro-Filho. 2003. Vertical stratification of small mammals in the Atlantic rain Forest of south-eastern Brazil. Journal of Tropical Ecology 19: 501-507.

Voss, R.S.; D.P. Lunde \& N.B. Simmons. 2001. The mammals of Paracou, French Guiana: a neotropical lowland rainforest fauna. Part 2: nonvolant species. Bulletin of the American Museum of Natural History 263: 1-236

ZAR, J.H. 1999. Biostatistical analysis. Rio de Janeiro, PrenticeHall, 663p.

Submitted: 03.IX.2008; Accepted: 09.VI.2009.

Editorial responsibility: Luciano M. Verdade 